\title{
ASYMPTOTIC CORRELATIONS BETWEEN ROTATED SOLUTIONS IN FACTOR ANALYSIS
}

\author{
Haruhiko Ogasawara*,1)
}

\begin{abstract}
The asymptotic correlations between differently rotated solutions in exploratory factor analysis are derived. The solutions are orthogonally or obliquely rotated for unstandardized or standardized manifest variables. To obtain the asymptotic correlations between different solutions, the covariance models for manifest variables have been constructed so that two sets of solutions are involved in a single covariance structure. The asymptotic correlations can be used for the statistical test of the differences of rotated solutions. The correlation matrix between the rotated factors of the first solution and those of the second is also introduced in the models with appropriate restrictions to identify the models. The asymptotic standard errors of the estimates of the correlations between the factors in different solutions are simultaneously provided. A numerical example is presented with simulated values.
\end{abstract}

\section{Introduction}

Factor analysis is one of the most frequently used methods of multivariate data analysis in behavioral and social sciences. While confirmatory factor analysis models as special cases of covariance structure models have been developed and well investigated in recent years, the statistical analyses for the results of exploratory factor analysis especially those with factor rotation have not sufficiently been provided in spite of the huge amount of its usage in practice.

The formulas for the asymptotic standard errors of rotated factor loadings have been developed by Archer and Jennrich (1973) (see also Jennrich, 1974) and Jennirich (1973a) for orthogonal and oblique models, respectively. The actual results required to apply the above formulas to individual rotation methods have been given, for example, by Archer and Jennrich (1973) for the raw orthomax method, Jennrich (1973a) for the raw-oblimin method, Ogasawara (1996; see also 1998a) for the orthomax method with Kaiser's normalization, Ogasawara (1998b) for the promax solution and Ogasawara (1999a) for the direct oblimin method with Kaiser's normalization. Methods using numerical derivatives for the asymptotic standard errors have been given by Browne and DuToit (1992) (see also Cudeck $\&$ O'Dell, 1994). For the standard errors of rotated loadings, Jennrich (1973b) showed statistically stable or unstable results caused by factor rotation, and called them the Wexler and anti-Wexler phenomena, respectively.

The purpose of this paper is to derive the asymptotic correlations among differently rotated solutions in factor analysis under the assumption of multivariate

Key Words and Phrases: factor rotation,asymptotic correlations,standard errors, information matrix, Kaiser's normalization.

${ }^{*}$ Department of Information and Management Science, Otaru University of Commerce, 35-21, Midori, Otaru 047-8501 Japan. E-rnail : hogasa@ires.otaru-uc.ac.jp

1) The author is indebted to an anonymous reviewer for helpful comments and suggestions. 
normality. The estimates of rotated loadings are subject to sampling variations. The standard errors of the loadings give information with respect to the statistical stability for individual solutions. However. they do not give the information as to the statistical relationships between different solutions. It is known that the normal varimax solution often gives results similar to those by the raw varimax method (the "normal" solution indicates the solution with Kaiser's normalization). From the statistical hypothesis testing. the problem becomes the test of the equal corresponding loadings in the two solutions. For the test, joint distribution of the corresponding estimated loadings in the two solutions is required. Since the two solutions are obtained from the same sample covariance/correlation matrices, one solution is not independent of the other solution. We will derive the asymptotic covariances/correlations between the two solutions, which give the asymptotic standard deviations for the differences of the corresponding estimated parameters in the two solutions.

The relationships between factors within a single solution or between differently rotated solutions can also be evaluated by factor correlations. If an orthogonal model is employed, the correlations between the orthogonal factors within a solution are always zero or one, while they are generally correlated with the factors in a differently rotated orthogonal solution. The estimates of the factor correlations are subject to sampling variations. The asymptotic standard errors of the estimates of the correlations will also be derived.

When exploratory factor analysis is used in behavioral sciences, manifest variables are usually standardized with unit variances. In this paper, for simplicity of model description. the models with unstandardized manifest variables will be given first. Then, the models for standardized manifest variables will be introduced based on the results for unstandardized variables. A numerical example will be presented with typical factor rotations.

\section{Two Orthogonally Rotated Solutions}

In this section, the results for two orthogonally rotated solutions are provided. Let $\boldsymbol{x}$ be the $p \times 1$ vector of random manifest variables and be described as:

$$
\boldsymbol{x}=\boldsymbol{\mu}+\Lambda f+e,
$$

where $E(\boldsymbol{x})=\boldsymbol{\mu}(p \times 1) ; E(\boldsymbol{f})=\mathbf{0}(q \times 1): \operatorname{Var}(\boldsymbol{f})=I_{q}$. where $I_{q}$ is the $q \times q$ identity matrix and $q$ is the number of common factors: $E(\boldsymbol{e})=\mathbf{0}(p \times 1)$; $\operatorname{Var}(\boldsymbol{e})=\Psi(p \times p)$ is a diagonal matrix: $\operatorname{Cov}(\boldsymbol{f}, \boldsymbol{e})=O(q \times p)$; and $\Lambda$ is a $p \times q$ factor loading matrix. From the model, we have the covariance matrix for $x$ :

$$
\Sigma=\Lambda \Lambda^{\prime}+\Psi \text {. }
$$

We suppose that the common factors are orthogonally rotated in two different methods. That is, the two rotated loading matrices are

$$
B_{1}=\Lambda T_{1}, T_{1}^{\prime} T_{1}=I_{q} \text { and } B_{2}=\Lambda T_{2}, T_{2}^{\prime} T_{2}=I_{q} .
$$


From (2) and (3), $\Sigma$ can be described as

$$
\Sigma=\Lambda T_{1} T_{1}^{\prime} T_{2} T_{2}^{\prime} \Lambda^{\prime}+\Psi=\Lambda T_{1} \Phi_{12} T_{2}^{\prime} \Lambda^{\prime}+\Psi=B_{1} \Phi_{12} B_{2}^{\prime}+\Psi,
$$

where $\Phi_{12}=E\left(T_{1}^{\prime} f f^{\prime} T_{2}\right)=T_{1}^{\prime} T_{2}$ is the inter-correlation matrix for the two differently rotated sets of factors $T_{1}^{\prime} f$ and $T_{2}^{\prime} f$. Since $B_{2}=\Lambda T_{2}=\Lambda T_{1} T_{1}^{\prime} T_{2}=$ $B_{1} \Phi_{12}, \Phi_{12}$ is a kind of rotation (transformation) matrix and (4) is similar to Ogasawara's (1998b, (7)) model. The difference is that in (4) two differently rotated solutions are considered while $B_{1}$ in Ogasawara (1998b) is a just identified confirmatory loading matrix which is not of direct interest in his model.

We are interested in the asymptotic correlations between the estimates $\hat{B}_{1}$ and $\hat{B}_{2}$, and the asymptotic standard errors for the estimates $\hat{\Phi}_{12}$. They are obtained from the information matrix with appropriate restrictions to identify the model of (4). Let $l$ be the $\log$ Wishart likelihood for $B_{1}, B_{2}$ and $\Phi_{12}$. Then,

$$
l=-(n / 2)\left\{\ln |\Sigma|+\operatorname{tr}\left(\Sigma^{-1} S\right)\right\}+\text { const. }
$$

where $\Sigma=B_{1} \Phi_{12} B_{2}^{\prime}+\Psi ; n=N^{+}-1 ; N$ is the number of observations and $S$ is an unbiased sample covariance matrix for manifest variables. Let $r=$ $\boldsymbol{r}\left(B_{1}, \Phi_{12}, B_{2}\right)=\mathbf{0}$ be the vector with an appropriate dimension for the restrictions for the parameters. The restrictions come partly from removing rotational indeterminacies and partly from the redundant expression in (4). Let $\theta$ be the vector whose elements are the non-fixed parameters in $B_{1}, B_{2}, \Phi_{12}$ and $\Psi$. Then, the asymptotic covariance matrix for the maximum likelihood estimators of the parameters is obtained from the submatrix $I^{*}$ of the inverse of the augmented information matrix $I_{A}$ (see e.g., Silvey, 1975):

$$
I_{A}^{-1}=\left[\begin{array}{cc}
I & \partial r^{\prime} / \partial \boldsymbol{\theta} \\
\partial r / \partial \boldsymbol{\theta}^{\prime} & O
\end{array}\right]^{-1}=\left[\begin{array}{cc}
I^{*} & \# \\
\# & \#
\end{array}\right]
$$

where $I$ is the information matrix, that is,

$$
(I)_{i j}=I\left(\theta_{i}, \theta_{j}\right)=E\left(-\partial^{2} l / \partial \theta_{i} \partial \theta_{j}\right)=\frac{n}{2} \operatorname{tr}\left(\Sigma^{-1} \frac{\partial \Sigma}{\partial \theta_{i}} \Sigma^{-1} \frac{\partial \Sigma}{\partial \theta_{j}}\right),
$$

in which $(\cdot)_{i j}$ denotes the $(i, j)$ th element of the matrix in parentheses; $\theta_{i}$ is the $i$-th element of $\boldsymbol{\theta}$; and \#'s indicate submatrices which are not used here. The asymptotic standard errors of the estimates are the square roots of the diagonal elements of $I^{*}$ and the asymptotic correlation matrix for the estimates of the parameters $\hat{B}_{1}, \hat{B}_{2}, \hat{\Phi}_{12}$ and $\hat{\Psi}$ is

$$
\operatorname{acor}(\hat{\boldsymbol{\theta}})=\left(\operatorname{Diag} I^{*}\right)^{-1 / 2} I^{*}\left(\operatorname{Diag} I^{*}\right)^{-1 / 2},
$$

where $\operatorname{Diag}(\cdot)$ denotes the diagonal matrix whose diagonal elements are those of the parenthesized matrix. By replacing the true values of the parameters in 
(6) and (8) by their estimates, we have the estimates of the asymptotic standard errors and correlations.

The restrictions $r=\mathbf{0}$ and consequently $I_{A}$ depend on rotation methods employed. However, the formula for the information matrix $I$, which will be shown in Appendix 1 (see (A1)), is common to rotated solutions. The first set of restrictions comes from the redundancy of having two orthogonally rotated loading-matrices in a single covariance structure. That is, from $B_{1} \Phi_{12}=B_{2}$ we have

$$
r_{1}=\operatorname{vec} R_{1}=\operatorname{vec}\left(B_{1} \Phi_{12}-B_{2}\right)=\mathbf{0},
$$

where $\boldsymbol{r}_{1}$ is a $p q \times 1$ vector and vec (.) operator vectorizes the parenthesized matrix by stacking each column of the matrix sequentially in a single column vector. The partial derivatives required in (6) are as follows:

$$
\begin{aligned}
& \frac{\partial r_{1 i j}}{\partial b_{1 s t}}=\delta_{i s} \phi_{12 t j}, \frac{\partial r_{1 i j}}{\partial b_{2 s t}}=-\delta_{i s} \delta_{j t}, \quad(i, s=1, \ldots, p ; j, t,=1, \ldots, q), \\
& \frac{\partial r_{1 i j}}{\partial \phi_{12 u v}}=\delta_{j v} b_{1 i u}, \quad(i=1, \ldots, p ; j, u, v=1, \ldots, q)
\end{aligned}
$$

where $r_{1 i j}=\left(R_{1}\right)_{i j}, b_{1 s t}=\left(B_{1}\right)_{s t}, b_{2 s t}=\left(B_{2}\right)_{s t}, \phi_{12 u v}=(\Phi)_{u v}$ and $\delta_{i s}$ is the Kronecker delta.

The second set of restrictions corresponds to the redundant parameters $\Phi_{12}$ in (4), though we are interested in this matrix. Noting that $\Phi_{12}$ is an orthogonal matrix, we have

$$
r_{2}=\operatorname{vech} R_{2}=\operatorname{vech}\left(\Phi_{12} \Phi_{21}-I_{p}\right)=\mathbf{0}
$$

where $\boldsymbol{r}_{2}$ is a $\left(q^{2}+q\right) / 2 \times 1$ vector; vech $(\cdot)$ denotes the vector consisting of the elements on or below the diagonal elements of the matrix in parentheses: and $\Phi_{21}=\Phi_{12}^{\prime}$. Since the number of the parameters in $\Phi_{12}$ is $q^{2}, r_{2}=0$ is not sufficient for removing the redundancy of introducing the matrix $\Phi_{12}$ in (4). This will be completed by one of the following third and fourth sets of restrictions (or the first set of restrictions). The partial derivatives of $\boldsymbol{r}_{2}$ with respect to $\Phi_{12}$ is

$$
\frac{\partial r_{2 i j}}{\partial \phi_{12 u v}}=\delta_{i u} \phi_{12 j v}+\delta_{j u} \phi_{12 i v},(q \geq i \geq j \geq 1: u, v=1, \ldots, q)
$$

where $r_{2 i j}=\left(R_{2}\right)_{i j}$.

The third and fourth sets of restrictions are concerned with rotational indeterminacies. Let $h_{1}=h_{1}\left(B_{1}\right)$ and $h_{2}=h_{2}\left(B_{2}\right)$ be analytical rotation criteria to be optimized by $B_{1}$ and $B_{2}$, respectively. Then, from Archer and Jennrich (1973), the third and fourth sets of restrictions are

$$
\boldsymbol{r}_{3}=\operatorname{vb} R_{3}=\mathrm{vb}\left(B_{1}^{\prime} \frac{\partial h_{1}}{\partial B_{1}}-\frac{\partial h_{1}}{\partial B_{1}^{\prime}} B_{1}\right)=\mathbf{0}
$$

and

$$
\boldsymbol{r}_{4}=\operatorname{vb} R_{4}=\operatorname{vb}\left(B_{2}^{\prime} \frac{\partial h_{2}}{\partial B_{2}}-\frac{\partial h_{2}}{\partial B_{2}^{\prime}} B_{2}\right)=\mathbf{0}
$$


respectively, where $\boldsymbol{r}_{3}$ and $\boldsymbol{r}_{4}$ are $\left(q^{2}-q\right) / 2 \times 1$ vectors and $\mathrm{vb}(\cdot)$ denotes the vector consisting of the elements below the diagonal elements of the matrix in parentheses. The partial derivatives of $h_{1}$ and $h_{2}$ with respect to $B_{1}$ and $B_{2}$ depend on the functions $h_{1}$ and $h_{2}$ (see the references in the previous section). The overall restrictions are summarized as

$$
r=\left(\boldsymbol{r}_{1}^{\prime}, \boldsymbol{r}_{2}^{\prime}, \boldsymbol{r}_{3}^{\prime}, \boldsymbol{r}_{4}^{\prime}\right)^{\prime}=\mathbf{0}
$$

Since the number of restrictions for $\boldsymbol{r}_{1}, \boldsymbol{r}_{2}, \boldsymbol{r}_{3}$ and $\boldsymbol{r}_{4}$ are $p q,\left(q^{2}+q\right) / 2,\left(q^{2}-q\right) / 2$ and $\left(q^{2}-q\right) / 2$, respectively, the total number of restrictions becomes

$$
n_{r}=p q+\left(3 q^{2}-q\right) / 2 .
$$

On the other hand, since the numbers of parameters in $B_{1}, B_{2}, \Phi_{12}$ and $\Psi$ are $p q$, $p q, q^{2}$ and $p$, respectively, the total number of parameters is

$$
n_{p}=2 p q+p+q^{2} .
$$

Consequently, the number of independent parameters is

$$
n_{p}-n_{r}=p q+p-\left(q^{2}-q\right) / 2,
$$

which is equal to the number of independent parameters in the usual exploratory factor analysis model (see (2)).

\section{An Orthogonally Rotated Solution and an Obliquely Rotated So- lution}

Next, we consider the relationships between an orthogonally rotated solution and an obliquely rotated solution. In this section the same notation $B_{2}$ is used for an obliquely rotated loading matrix as that for the orthogonally rotated solution to avoid complicated notations. In addition to the parameters in the previous section we have to consider the correlations within the second set of obliquely rotated factors. Considering all parameters concerned with the two sets of factors, the covariance structure can be described as:

$$
\begin{aligned}
\Sigma & =\Lambda T_{1} T_{1}^{\prime} T_{2} T_{2}^{-1} \Lambda^{\prime}+\Psi=\Lambda T_{1} \Phi_{12} T_{2}^{-1} \Lambda^{\prime}+\Psi \\
& =B_{1} \Phi_{12} B_{2}^{\prime}+\Psi=B_{1} \Phi_{12} \Phi_{22}^{-1} \Phi_{21} \Phi_{12} B_{2}^{\prime}+\Psi
\end{aligned}
$$

with Diag $\Phi_{22}=I_{q}$ where $T_{1}$ is the same as in (4): $T_{2}$ is the rotation matrix with Diag $\left(T_{2}^{\prime} T_{2}\right)=I_{q}$; and $\Phi_{22}=T_{2}^{\prime} T_{2}$ is the correlation matrix for the second set of oblique factors with unit variances. Let $\theta$ be the vector consisting of the non-duplicated non-fixed parameters in $B_{1}, B_{2}, \Phi_{12}, \Phi_{22}$ and $\Psi$. Then, the $\log$ Wishart likelihood and the augmented information matrix are obtained with $\Sigma=$ 
$B_{1} \Phi_{12} \Phi_{22}^{-1} \Phi_{21} \Phi_{12} B_{2}^{\prime}+\Psi$ similarly to the case of two orthogonal solutions (see (5) and (6)). The information matrix will be given in Appendix 1 (see (A2)).

The restrictions for model identification are obtained in the following way. Noting that $B_{1} \Phi_{12}=B_{2} \Phi_{22}$, the first set of restrictions is

$$
\boldsymbol{r}_{1}=\operatorname{vec} R_{1}=\operatorname{vec}\left(B_{1} \Phi_{12}-B_{2} \Phi_{22}\right)=\mathbf{0}
$$

where $\boldsymbol{r}_{1}$ is a $p q \times 1$ vector. The partial derivatives with respect to the parameters are

$$
\begin{aligned}
\frac{\partial r_{1 i j}}{\partial b_{1 s t}} & =\delta_{i s} \phi_{12 t j}, \frac{\partial r_{1 i j}}{\partial b_{2 s t}}=-\delta_{i s} \phi_{22 t j},(i, s=1, \ldots, p ; j, t=1, \ldots, q), \\
\frac{\partial r_{1 i j}}{\partial \phi_{12 u v}} & =\delta_{j v} b_{1 i u},(i=1, \ldots, p ; j, u, v=1, \ldots, q), \\
\frac{\partial r_{1 i j}}{\partial \phi_{22 u v}} & =-\delta_{j v} b_{2 i u}-\delta_{j u} b_{2 i v},(i=1, \ldots, p ; j=1, \ldots, q ; q \geq u>v \geq 1),
\end{aligned}
$$

where $\phi_{22 u v}=\left(\Phi_{22}\right)_{u v}$.

The second set of restrictions are concerned with $\Phi_{12}$ and $\Phi_{22}$. Note that $\Phi_{12}$ is no longer a transformation matrix from a loading matrix to a different loading matrix. However, from (19) we see that $\Phi_{12}$ is a transformation matrix from the loading matrix $B_{1}$ to the structure matrix of the second set of factors $B_{2} \Phi_{22}$. Since $\Phi_{12}=T_{1}^{\prime} T_{2}$ and $\Phi_{22}=T_{2}^{\prime} T_{2}$, we have

$$
\boldsymbol{r}_{2}=\operatorname{vech} R_{2}=\operatorname{vech}\left(\Phi_{12} \Phi_{22}^{-1} \Phi_{21}-I_{q}\right)=\mathbf{0}
$$

where $\boldsymbol{r}_{2}$ is a $\left(q^{2}+q\right) / 2 \times 1$ vector. The partial derivatives of $\boldsymbol{r}_{2}$ with respect to the parameters are

$$
\begin{aligned}
\frac{\partial r_{2 i j}}{\partial \phi_{12 u v}}= & \delta_{i u}\left(\Phi_{12} \Phi_{22}^{-1}\right)_{j v}+\delta_{j u}\left(\Phi_{12} \Phi_{22}^{-1}\right)_{i v},(q \geq i \geq j \geq 1 ; u, v=1, \ldots, q) \\
\frac{\partial r_{2 i j}}{\partial \phi_{22 u v}}= & -\left(\Phi_{12} \Phi_{22}^{-1}\left(I_{u v}+I_{v u}\right) \Phi_{22}^{-1} \Phi_{21}\right)_{i j} \\
= & -\left(\Phi_{12} \Phi_{22}^{-1}\right)_{i u}\left(\Phi_{12} \Phi_{22}^{-1}\right)_{j v}-\left(\Phi_{12} \Phi_{22}^{-1}\right)_{i v}\left(\Phi_{12} \Phi_{22}^{-1}\right)_{j u} \\
& (q \geq i \geq j \geq 1 ; q \geq u>v \geq 1)
\end{aligned}
$$

The third set of restriction is the same as (13a):

$$
\boldsymbol{r}_{3}=\operatorname{vb}\left(B_{1}^{\prime} \frac{\partial h_{1}}{\partial B_{1}}-\frac{\partial h_{1}}{\partial B_{1}^{\prime}} B_{1}\right)=\mathbf{0}
$$

where $r_{3}$ is a $\left(q^{2}-q\right) / 2 \times 1$ vector and depends on the forms of $h_{1}$. The fourth set of restrictions are concerned with the rotational indeterminacy for $B_{2}$ and is described as:

$$
\boldsymbol{r}_{4}=\operatorname{vo}\left(B_{2}^{\prime} \frac{\partial h_{2}}{\partial B_{2}} \Phi_{22}^{-1}\right)=\mathbf{0}
$$


(see, Jennrich, 1973a), where vo $(\cdot)$ vectorizes all off-diagonal elements of the parenthesized matrix and hence $\boldsymbol{r}_{4}$ is a $\left(q^{2}-q\right) \times 1$ vector. For the actual derivatives of (24) with respect to the associated parameters, see the references in the introductory section.

The number of the overall restrictions $\boldsymbol{r}=\left(\boldsymbol{r}_{1}^{\prime}, \boldsymbol{r}_{2}^{\prime}, \boldsymbol{r}_{3}^{\prime}, \boldsymbol{r}_{4}^{\prime}\right)^{\prime}=\mathbf{0}$ is

$$
n_{r}=p q+\left(q^{2}+q\right) / 2+\left(q^{2}-q\right) / 2+\left(q^{2}-q\right)=p q+2 q^{2}-q,
$$

while the total number of parameters in $B_{1}, B_{2}, \Phi_{12}, \Phi_{22}$ and $\Psi$ is

$$
n_{p}=p q+p q+q^{2}+\left(q^{2}-q\right) / 2+p=2 p q+p+\left(3 q^{2}-q\right) / 2
$$

Consequently, we see that the number of independent parameters $n_{p}-n_{r}=$ $p q+p-\left(q^{2}-q\right) / 2$ is unchanged.

\section{Two Obliquely Rotated Solutions}

In this section, the results for two obliquely rotated solutions are provided. The additional parameters other than those in the previous section are the nonduplicated off-diagonal elements of $\Phi_{11}$, the correlation matrix between the oblique factors in the first solution. Let $T_{1}$ and $T_{2}$ be the rotation matrices with $\operatorname{Diag}\left(T_{1}^{\prime} T_{1}\right)=$ $\operatorname{Diag}\left(T_{2}^{\prime} T_{2}\right)=I_{q}$. Then, the covariance matrix of the manifest variables is described as:

$$
\begin{aligned}
\Sigma & =\Lambda T_{1}^{\prime-1} T_{1}^{\prime} T_{2} T_{2}^{-1} \Lambda^{\prime}+\Psi=\Lambda T_{1}^{\prime-1} \Phi_{12} T_{2}^{-1} \Lambda^{\prime}+\Psi \\
& =B_{1} \Phi_{12} B_{2}^{\prime}+\Psi=B_{1} \Phi_{12} \Phi_{22}^{-1} \Phi_{21} \Phi_{11}^{-1} \Phi_{12} B_{2}^{\prime}+\Psi
\end{aligned}
$$

with $T_{1}^{\prime} T_{1}=\Phi_{11}, \operatorname{Diag} \Phi_{11}=I_{q}, T_{2}^{\prime} T_{2}=\Phi_{22}$ and $\operatorname{Diag} \Phi_{22}=I_{q}$, where the same notation $B_{1}$ as in the previous sections is used for the oblique solution in this section. The information matrix for the non-duplicated non-fixed parameters in $B_{1}, B_{2}, \Phi_{12}, \Phi_{11}, \Phi_{22}$ and $\Psi$ will be given in Appendix 1 (see (A3)).

The restrictions are obtained as follows. The first set of restrictions is

$$
\boldsymbol{r}_{1}=\operatorname{vec} R_{1}=\operatorname{vec}\left(B_{1} \Phi_{12}-B_{2} \Phi_{22}\right)=\mathbf{0},
$$

where $\boldsymbol{r}_{1}$ is a $p q \times 1$ vector and equal to (19) in form. The partial derivatives with respect to the parameters are the same as (20). Noting that $\Phi_{11}=T_{1}^{\prime} T_{1}$ and $\Phi_{22}=T_{2}^{\prime} T_{2}$, the second set of restrictions is

$$
r_{2}=\operatorname{vech} R_{2}=\operatorname{vech}\left(\Phi_{11}-\Phi_{12} \Phi_{22}^{-1} \Phi_{21}\right)=0
$$

where $\boldsymbol{r}_{2}$ is a $\left(q^{2}+q\right) / 2 \times 1$ vector. The partial derivatives of $\boldsymbol{r}_{2}$ with respect to the associated parameters are

$$
\frac{\partial r_{2 i j}}{\partial \phi_{12 u v}}=-\delta_{i u}\left(\Phi_{12} \Phi_{22}^{-1}\right)_{j v}-\delta_{j u}\left(\Phi_{12} \Phi_{22}^{-1}\right)_{i v},(q \geq i \geq j \geq 1 ; u, v=1, \ldots, q),
$$




$$
\begin{aligned}
\frac{\partial r_{2 i j}}{\partial \phi_{11 u v}}= & \delta_{i u} \delta_{j v}, \\
\frac{\partial r_{2 i j}}{\partial \phi_{22 u v}}= & \left(\Phi_{12} \Phi_{22}^{-1}\right)_{i u}\left(\Phi_{12} \Phi_{22}^{-1}\right)_{j v}+\left(\Phi_{12} \Phi_{22}^{-1}\right)_{i v}\left(\Phi_{12} \Phi_{22}^{-1}\right)_{j u} \\
& (q \geq i \geq j \geq 1 ; q \geq u>v \geq 1),
\end{aligned}
$$

where $\phi_{11 u v}=\left(\Phi_{11}\right)_{u v}$. The third and fourth sets of restrictions are similar to $(23)$ :

$$
\boldsymbol{r}_{3}=\mathrm{vo}\left(B_{1}^{\prime} \frac{\partial h_{1}}{\partial B_{1}} \Phi_{11}^{-1}\right)=\mathbf{0} \text { and } \boldsymbol{r}_{4}=\text { vo }\left(B_{2}^{\prime} \frac{\partial h_{2}}{\partial B_{2}} \Phi_{22}^{-1}\right)=\mathbf{0}
$$

where $h_{1}=h_{1}\left(B_{1}\right)$ in this section is an oblique rotation criterion to be optimized by $B_{1}$. The dimensions of $\boldsymbol{r}_{3}$ and $\boldsymbol{r}_{4}$ are each $q^{2}-q$.

The number of the overall restrictions $r=\left(\boldsymbol{r}_{1}^{\prime}, \boldsymbol{r}_{2}^{\prime}, \boldsymbol{r}_{3}^{\prime}, \boldsymbol{r}^{\prime}\right)^{\prime}=\mathbf{0}$ is

$$
n_{r}=p q+\left(q^{2}+q\right) / 2+q^{2}-q+q^{2}-q=p q+\left(5 q^{2}-3 q\right) / 2,
$$

while the total number of the parameters in $B_{1}, B_{2}, \Phi_{12}, \Phi_{11}, \Phi_{22}$ and $\Psi$ is

$$
\begin{aligned}
n_{p} & =p q+p q+q^{2}+\left(q^{2}-q\right) / 2+\left(q^{2}-q\right) / 2+p \\
& =2 p q+p+2 q^{2}-q
\end{aligned}
$$

which gives the unchanged number of the independent parameters $n_{p}-n_{r}=$ $p q+p-\left(q^{2}-q\right) / 2$.

\section{Models for Standardized Manifest Variables}

The models for standardized manifest variables for orthogonal and/or oblique factors can be obtained by replacing $\Sigma$ in (4), (18) and (27) with the population correlation matrix $\mathrm{P}$ for manifest variables. Let $D=\operatorname{diag}\left(d_{1}, \ldots, d_{p}\right)$ be the diagonal matrix with the $i$-th diagonal element $d_{i},(i=1, \ldots, p)$ being the standard deviation of the $i$-th unstandardized manifest variable. Then,

$$
\Sigma=D P D
$$

and the covariance matrices for standardized manifest variables corresponding to (4), (18) and (27) are

$$
\begin{aligned}
& \Sigma=D\left(B_{1} \Phi_{12} B_{2}^{\prime}+\Psi\right) D \\
& \Sigma=D\left(B_{1} \Phi_{12} \Phi_{22}^{-1} \Phi_{21} \Phi_{12} B_{2}^{\prime}+\Psi\right) D \\
& \Sigma=D\left(B_{1} \Phi_{12} \Phi_{22}^{-1} \Phi_{21} \Phi_{11}^{-1} \Phi_{12} B_{2}^{\prime}+\Psi\right) D
\end{aligned}
$$

with $\operatorname{DiagP}=I_{p}$ and $\operatorname{Diag} \Phi_{11}=\operatorname{Diag} \Phi_{22}=I_{q}$

The information matrices for the models of (35) will be given in Appendix 1 (see (A4)). All of the restrictions described in the previous sections should be 
imposed on the corresponding parameters in (35). Since $\mathrm{P}$ is a correlation matrix, we have $p$ additional restrictions for each of the models of (35). That is,

$$
\boldsymbol{r}_{5}=(\operatorname{DiagP}) \mathbf{1}_{p}-\mathbf{1}_{p}=\left\{\operatorname{Diag}\left(B_{1} \Phi_{12} B_{2}^{\prime}+\Psi\right)\right\} \mathbf{1}_{p}-\mathbf{1}_{p}=\mathbf{0},
$$

where $\boldsymbol{r}_{5}$ is a $p \times 1$ vector and $\mathbf{1}_{p}$ is the $p \times 1$ vector consisting of ones. Note that (36) holds not only for (35a) but also for (35b) and (35c). For (35b) the restrictions $\Phi_{12} \Phi_{22}^{-1} \Phi_{21}=I_{q}$ have already been imposed by (21) and the restrictions can be substituted for (35b), which yields (36). Similarly, for $(35 \mathrm{c})$, the restrictions $\Phi_{11}=\Phi_{12} \Phi_{22}^{-1} \Phi_{21}$ (see (29)) can be substituted for (35c), which gives (36). The common partial derivatives of $\boldsymbol{r}_{5}$ with respect to the parameters are

$$
\begin{aligned}
\frac{\partial \rho_{i i}}{\partial b_{1 s t}} & =\delta_{i s}\left(B_{2} \Phi_{21}\right)_{i t}, \frac{\partial \rho_{i i}}{\partial b_{2 s t}}=\delta_{i s}\left(B_{1} \Phi_{12}\right)_{i t},(i, s=1, \ldots, p ; t=1, \ldots, q), \\
\frac{\partial \rho_{i i}}{\partial \phi_{12 u v}} & =b_{1 i u} b_{2 i v},(i=1, \ldots, p ; u, v=1, \ldots, q), \\
\frac{\partial \rho_{i i}}{\partial \psi_{s}} & =\delta_{i s},(i, s=1, \ldots, p),
\end{aligned}
$$

where $\rho_{i i}=(\mathrm{P})_{i i}$ and $\psi_{s}=(\Psi)_{s s}$. The number of the additional restrictions in (36) for each model for standardized variables is $p$, while the additional parameters are $d_{1}, \ldots, d_{p}$ whose number is $p$. Hence, the independent numbers of parameters are unchanged by standardization of manifest variables.

\section{A Numerical Example}

A numerical example based on the correlation matrix for eight physical variables ( $N=305$; Harman, 1976, p.22) will be presented. Table 1 shows six rotated solutions with the assumption of a two-factor model: the principal factor solution, the raw- and normal-varimax solutions, the raw- and normal-direct quartimin solutions, and the normal oblique varimax solution (Crawford, 1975; Browne \& DuToit, 1992). The principal factor solution $\left(B^{\prime} B\right.$ : diagonal) is a kind of maximum likelihood solution, which is obtained from the spectral decomposition of $\Lambda \Lambda^{\prime}$.

Table 2 gives the theoretical estimates of the asymptotic correlations between the estimated loadings for Factors I of the principal factor- and normal varimax solutions for standardized variables. Table 2 also shows the corresponding simulated values, which have been obtained in the following way. First, the fitted correlation matrix by the two-factor model was regarded as a population variance-covariance matrix. Based on the population covariance matrix, random observations of the sample size equal to the real one $(N=305)$ were generated under the assumption of multivariate normality. From the generated data, the parameters were estimated with factor rotation. For a rotated solution, we still have the indeterminacy of the signs and permutations in the columns of a rotated loading matrix, which has 
Table 1: Estimated loadings for the eight physical variables

\begin{tabular}{|c|c|c|c|c|c|c|}
\hline \multirow{2}{*}{$\begin{array}{l}\text { Varia- } \\
\text { ble } \\
\text { No. }\end{array}$} & \multicolumn{2}{|c|}{$\begin{array}{l}\text { Principal } \\
\text { factor }\end{array}$} & \multicolumn{2}{|c|}{$\begin{array}{c}\text { Raw } \\
\text { varimax }\end{array}$} & \multicolumn{2}{|c|}{$\begin{array}{l}\text { Normal } \\
\text { varimax }\end{array}$} \\
\hline & I & II & I & II & I & II \\
\hline 1 & .86 & .31 & .871 & .267 & .863 & .293 \\
\hline 2 & .84 & .43 & .931 & .159 & .926 & .187 \\
\hline 3 & .82 & 41 & .899 & .158 & .894 & .185 \\
\hline 4 & .83 & .33 & .864 & .232 & .857 & .258 \\
\hline 5 & .75 & -.59 & .254 & .920 & .227 & .927 \\
\hline 6 & .63 & -.49 & .212 & .769 & .189 & .775 \\
\hline 7 & .57 & -.51 & .151 & .749 & .129 & .753 \\
\hline 8 & .60 & -.32 & .292 & .615 & .273 & .623 \\
\hline $\begin{array}{l}\text { Varia- } \\
\text { ble }\end{array}$ & \multicolumn{2}{|c|}{$\begin{array}{c}\text { Raw } \\
\text { quartimin }\end{array}$} & \multicolumn{2}{|c|}{$\begin{array}{c}\text { Normal } \\
\text { quartimin }\end{array}$} & \multicolumn{2}{|c|}{$\begin{array}{l}\text { Normal } \\
\text { oblique } \\
\text { varimax }\end{array}$} \\
\hline No. & I & II & I & II & I & II \\
\hline 1 & .869 & .084 & .869 & .083 & .85 & .14 \\
\hline 2 & .967 & -.049 & .968 & -.050 & .94 & .21 \\
\hline 3 & .932 & -.042 & .933 & -.044 & .90 & .25 \\
\hline 4 & .872 & .047 & .872 & .046 & .85 & .11 \\
\hline 5 & .005 & .952 & -.007 & .958 & .06 & .93 \\
\hline 6 & .004 & .796 & -.006 & .801 & .05 & .78 \\
\hline 7 & -.057 & .789 & -.066 & .793 & -.01 & .77 \\
\hline 8 & .136 & .607 & .129 & .610 & .17 & .60 \\
\hline$\hat{\phi_{21}}$ & \multicolumn{2}{|c|}{.463} & \multicolumn{2}{|c|}{.473} & \multicolumn{2}{|c|}{.36} \\
\hline
\end{tabular}

been removed by selecting the solution which is closest to the population loadings. The estimation was repeated 1,000 times and we had 1,000 estimates for each parameter. The simulated correlations are the correlations computed from these values. The simulated values in Table 2 are close to the corresponding theoretical values.

Table 3 shows the theoretical asymptotic correlations for the five combinations (A-E) of orthogonal and/or oblique solutions. The values are for the corresponding parameters for two solutions. That is, for the combination $A$, the values for Factors I for standardized variables are the diagonal elements of the theoretical asymptotic correlation matrix in Table 2. The value acor $\left(\hat{\phi}_{1121}, \hat{\phi}_{22} 21\right)$ in Table 3 is the estimate of the asymptotic correlation between $\left(\hat{\Phi}_{11}\right)_{21}=\hat{\phi}_{1121}$ and $\left(\hat{\Phi}_{22}\right)_{21}=\hat{\phi}_{22} 21$. The results in Table 3 include those for unstandardized variables, which have been obtained by regarding the sample correlation matrix as a sample variance-covariance matrix. From the table we see that when two solutions are rather different (e.g., A), the absolute values of the correlations between them tend to be small and the correlations can be negative. In contrast, the correlations become very high when two solutions are similar ( $\mathrm{B}, \mathrm{D}$ and $\mathrm{E})$. 
Table 2: Correlations between the estimated loadings for the eight physical variables (standardized variables)

\begin{tabular}{|c|c|c|c|c|c|c|c|c|c|}
\hline \multicolumn{10}{|c|}{$\begin{array}{l}\text { (1) Theoretical values of the asymptotic correlations } \\
\text { Principal factor solution: Factor I }\end{array}$} \\
\hline & & 1 & 2 & 3 & 4 & 5 & 6 & 7 & 8 \\
\hline \multirow{5}{*}{$\begin{array}{l}\text { Normal } \\
\text { varimax } \\
\text { solution: }\end{array}$} & 1 & .22 & .13 & .18 & .16 & -.32 & -.28 & -.31 & -.15 \\
\hline & 2 & -.01 & .10 & .01 & .02 & -.33 & -.27 & -.29 & -.16 \\
\hline & 3 & .13 & .10 & .27 & .15 & -.27 & -.22 & -.24 & -.11 \\
\hline & 4 & .18 & .17 & .21 & .31 & -.28 & -.24 & -.26 & -.12 \\
\hline & 5 & .25 & .26 & .27 & .26 & .83 & .42 & 34 & 35 \\
\hline \multirow[t]{5}{*}{ Factor I } & 6 & .20 & .22 & .21 & .21 & .47 & .83 & .23 & .24 \\
\hline & 7 & .15 & .15 & .15 & .15 & .49 & .31 & .85 & .25 \\
\hline & 8 & .23 & .26 & .25 & .23 & .24 & .14 & .10 & .76 \\
\hline & \multicolumn{9}{|c|}{$\begin{array}{c}\text { (2) Simulated values of the correlations } \\
\text { Principal factor solution: Factor I }\end{array}$} \\
\hline & & 1 & 2 & 3 & 4 & 5 & 6 & 7 & 8 \\
\hline \multirow{5}{*}{$\begin{array}{l}\text { Normal } \\
\text { várimax } \\
\text { solution: }\end{array}$} & 1 & .24 & .14 & .20 & .18 & -.32 & -.25 & -.29 & -.19 \\
\hline & 2 & .01 & .12 & -.01 & .03 & -.30 & -.28 & -.25 & -.21 \\
\hline & 3 & .16 & .08 & .28 & .12 & -.26 & -.19 & -.25 & -.11 \\
\hline & 4 & .19 & .13 & .22 & .34 & -.26 & -.18 & -.22 & -.08 \\
\hline & 5 & .23 & .28 & .27 & .26 & .81 & .41 & 35 & 34 \\
\hline \multirow[t]{3}{*}{ Factor I } & 6 & .16 & .18 & .19 & .21 & .44 & .81 & .21 & .24 \\
\hline & 7 & .14 & .17 & .15 & .11 & .51 & .30 & .86 & .27 \\
\hline & 8 & .24 & .21 & .24 & .26 & .26 & .15 & .10 & .75 \\
\hline
\end{tabular}

The correlations between the orthogonal and oblique solutions are moderate or high in this case (C). The value (.99) of acor $\left(\hat{\phi}_{1121}, \hat{\phi}_{22} 21\right)$ for the combination $\mathrm{E}$ is common to the cases of unstandardized and standardized variables, which comes from the property of normal solutions described in Appendix 2.

Table 4 gives the results of the tests of the equalities between two corresponding factor loadings (factor correlations). These are based on the asymptotic normality of the maximum likelihood estimators. The $z$-scores are obtained from, e.g.,

$$
\begin{aligned}
& z_{i j}=\frac{\hat{b}_{1 i j}-\hat{b}_{2 i j}}{\left\{\operatorname{ase}\left(\hat{b}_{1 i j}\right)^{2}+\text { ase }\left(\hat{b}_{2 i j}\right)^{2}-2 \operatorname{ase}\left(\hat{b}_{1 i j}\right) \text { aŝe }\left(\hat{b}_{2 i j}\right) \text { acor }\left(\hat{b}_{1 i j}, \hat{\mathrm{b}}_{2 i j}\right)\right\}^{1 / 2}}, \\
& (i=1, \ldots, p ; j=1, \ldots, q),
\end{aligned}
$$

which is asymptotically distributed according to the standardized normal distribution when $b_{1 i j}=b_{2 i j}$. The chi-squares in Table 4 are obtained in the following way. Let $\boldsymbol{b}_{1(i)}$ and $\boldsymbol{b}_{2(i)}$ be the $i$-th columns of $B_{1}$ and $B_{2}$, respectively. Then, it can be shown that

$$
\chi^{2}=\left(\hat{\boldsymbol{b}}_{1(i)}-\hat{\boldsymbol{b}}_{2(i)}\right)^{\prime}\left\{\widehat{\operatorname{acov}}\left(\hat{\boldsymbol{b}}_{1(i)}-\hat{\boldsymbol{b}}_{2(i)}\right)\right\}^{-1}\left(\hat{\boldsymbol{b}}_{1(i)}-\hat{\boldsymbol{b}}_{2(i)}\right)
$$


is asymptotically chi-square distributed with $d f=p$, when $\boldsymbol{b}_{1(i)}=\boldsymbol{b}_{2(i)}$. The matrix $\operatorname{arov}(\cdot)$ in (39) is the estimate of

$$
\begin{aligned}
\operatorname{acov}\left(\hat{b}_{1(i)}-\hat{\boldsymbol{b}}_{2(i)}\right)= & \operatorname{acov}\left(\hat{\boldsymbol{b}}_{1(i)}\right)+\operatorname{acov}\left(\hat{\boldsymbol{b}}_{2(i)}\right) \\
& -\operatorname{acov}\left(\hat{\boldsymbol{b}}_{1(i)}, \hat{\boldsymbol{b}}_{2(i)}\right)-\operatorname{acov}\left(\hat{\boldsymbol{b}}_{2(i)}, \hat{\boldsymbol{b}}_{1(i)}\right), \\
(i=1, \ldots, q) &
\end{aligned}
$$

where acov $\left(\hat{\boldsymbol{b}}_{1(i)}, \hat{\boldsymbol{b}}_{2(i)}\right)$ is a $p \times p$ asymptotic covariance matrix of $\hat{\boldsymbol{b}}_{1(i)}$ with respect to $\hat{\boldsymbol{b}}_{2(i)}$.

In Table 4, we find that the normal and raw solutions by the same rotation methods ( $B, D)$ do not show significant differences except for the case of the standardized variables for $\mathrm{B}$. This corresponds to the similarities of the raw and normal solutions shown in Table 1 . Notice that the normal oblique varimax and the normal/raw quartimin solutions in Table 1 are similar in a practical sense with slight advantage in terms of a simple structure for the quartimin solutions over the normal oblique varimax solution, while the statistical test for $\mathrm{E}$ shows significant differences. This means that the difference is small but it is statistically stable. The difference between the factor correlations (.473 vs. .36) may not be small even in the practical sense, while $\mathrm{z}=-11.2$ for this difference. (The value of $\mathrm{z}=-11.2$ for $\hat{\phi}_{1121}-\hat{\phi}_{2221}$ in $\mathrm{E}$ is common to the cases of unstandardized and standardized variables, which comes from the results shown in Appendix 2.)

In Table 4, we see that the absolute values of $z$ and chi-squares for the cases of standardized variables tend to be greater than the corresponding values for the cases of unstandardized variables. These tendencies are associated with the relatively small standard errors of parameter estimates for standardized variables (not shown here), which gives larger absolute values of $z$ (see (38)) on condition that the variances of unstandardized variables are set at unities for comparison and that the asymptotic correlations between parameter estimates concerned are on the same level.

Table 5 shows the estimates of the correlations between two sets of rotated factors with their corresponding standard errors. The simulated values in Table 5 have been obtained from the standard deviations of the 1,000 estimates of each parameter in the simulation mentioned earlier. The theoretical standard errors are close to their corresponding simulated values except for the cases with very small values (e.g., .000003). Since the results for the two sets of normal solutions (C and E) for unstandardized variables are equivalent to those for standardized variables, only one of the two sets are shown. This is a general result and will be explained in Appendix 2. The values of the correlations between the corresponding factors (the diagonal elements of $\hat{\Phi}_{12}$ ) for similar solutions are very high (B, D and E) and are accompanied by small standard errors. The absolute values of correlations must be less than or equal to one. Hence, the confidence interval for a correlation $\left(\Phi_{12}\right)_{i j}$ may violate this restriction. For this case, a variable transformation such 
Table 3: Asymptotic correlations between the estimated loadings and factor correlations for the eight physical variables

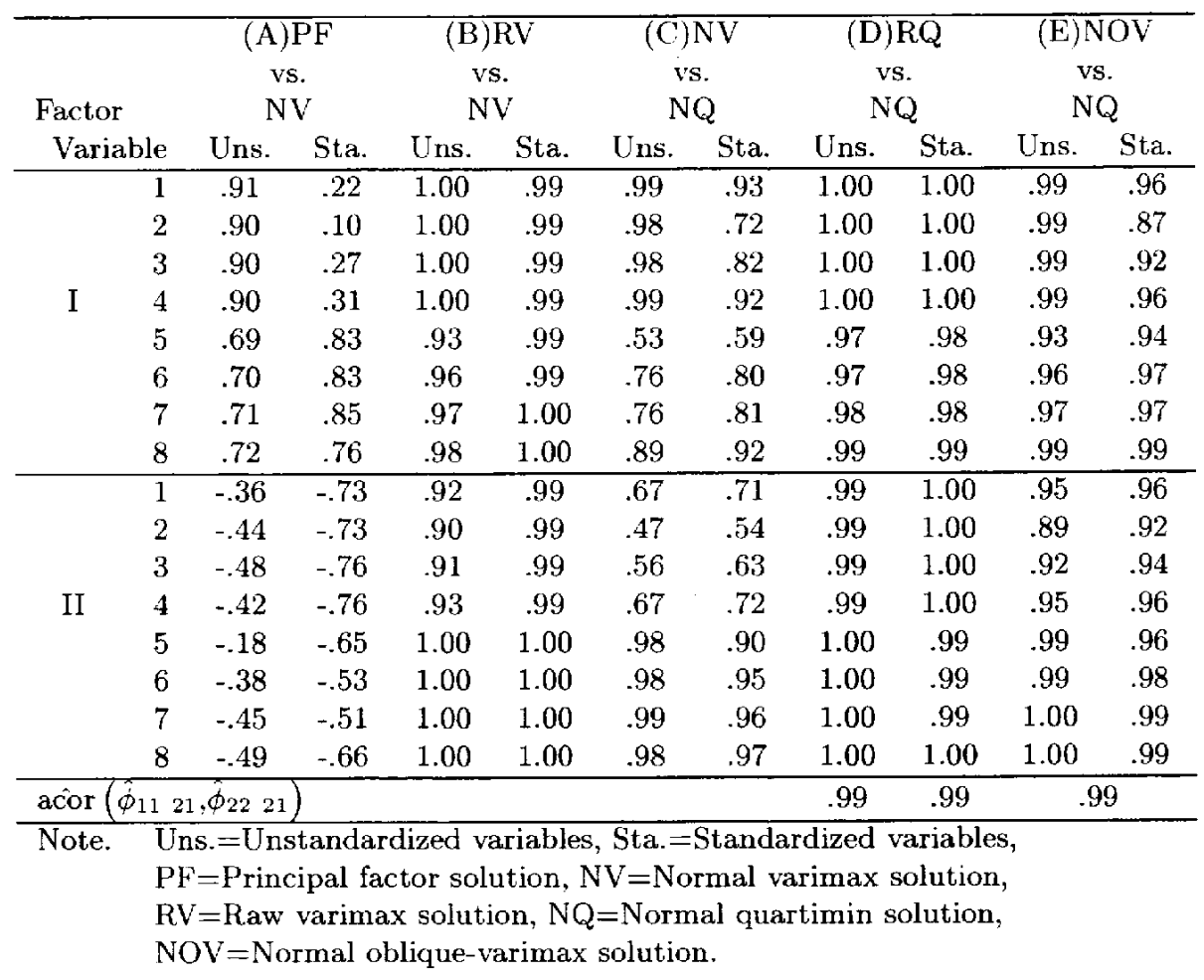

as Fisher's z-transformation can be used (see Browne, 1982). For the results of Table 5, the transformation does not seem to be needed. The standard errors in Table 5 are rather small $(<.05)$.

\section{Discussion}

Since the covariance structure models for manifest variables in our methods include two rotated solutions, the sizes of the information matrices tend to be large. The size of an augmented information matrix is $n_{r}+n_{p}$. The largest one is for the model for two oblique solutions for standardized variables. Noting that additional $p$ parameters and $p$ restrictions are needed for the models for standardized variables. the size becomes (see (32) and (33))

$$
\begin{aligned}
n_{r}+n_{p} & =\left\{p q-\left(5 q^{2}-3 q\right) / 2\right\}+\left(2 p q+p+2 q^{2}-q\right)+2 p \\
& =3 p q+3 p+\left(9 q^{2}-5 q\right) / 2 .
\end{aligned}
$$


Table 4: Tests of the equalities of the correspondeing loadings and factor correlations for eight physical variables

\begin{tabular}{|c|c|c|c|c|c|c|c|c|c|c|c|}
\hline \multirow{3}{*}{\multicolumn{2}{|c|}{$\begin{array}{l}\text { Factor } \\
\text { Variable }\end{array}$}} & \multirow{2}{*}{\multicolumn{2}{|c|}{$\begin{array}{l}\text { (A)PF } \\
\text { vs. } \\
\text { NV }\end{array}$}} & \multirow{2}{*}{\multicolumn{2}{|c|}{$\begin{array}{l}\text { (B)RV } \\
\text { vs. } \\
\text { NV }\end{array}$}} & \multirow{2}{*}{\multicolumn{2}{|c|}{$\begin{array}{l}\text { (C)NV } \\
\text { rs. } \\
\text { NQ }\end{array}$}} & \multirow{2}{*}{\multicolumn{2}{|c|}{$\begin{array}{c}\text { (D)RQ } \\
\text { vs. } \\
\text { NQ }\end{array}$}} & \multirow{2}{*}{\multicolumn{2}{|c|}{$\begin{array}{c}\text { (E) NOV } \\
\text { vs. } \\
\text { NQ }\end{array}$}} \\
\hline & & & & & & & & & & & \\
\hline & & Uns. & Sta. & Uns. & Sta. & Uns. & Sta. & Uns. & Sta. & Uns. & Sta. \\
\hline & 1 & -.2 & -.3 & 1.9 & 4.0 & -.8 & -.8 & .4 & .5 & -3.3 & -3.5 \\
\hline & 2 & -3.3 & -4.7 & 1.7 & 3.1 & -4.2 & -4.4 & -.6 & -.9 & -4.3 & -4.6 \\
\hline$z$ & 3 & -3.1 & -4.1 & 1.7 & 3.1 & -4.1 & -4.3 & -.6 & -1.0 & -4.4 & -4.6 \\
\hline for & 4 & -1.1 & -1.3 & 1.8 & 3.8 & -1.8 & -1.8 & .1 & .2 & -3.6 & -3.9 \\
\hline \multirow[t]{4}{*}{ I } & 5 & 11.1 & 29.3 & 2.1 & 5.2 & 7.4 & 8.5 & 1.2 & 1.3 & 5.8 & 6.3 \\
\hline & 6 & 9.7 & 19.5 & 2.1 & 5.5 & 7.4 & 8.5 & 1.2 & 1.4 & 5.8 & 6.3 \\
\hline & 7 & 9.4 & 19.0 & 2.1 & 5.5 & 7.4 & 8.4 & 1.2 & 1.4 & 6.2 & 6.8 \\
\hline & 8 & 8.0 & 11.3 & 2.1 & 5.7 & 6.6 & 7.5 & 1.3 & 1.4 & 4.3 & 4.5 \\
\hline \multirow[t]{5}{*}{$x^{2}$} & & 196 & 1238 & 5.5 & 64 & 121 & 1096 & 2.5 & 3.3 & 48 & 111 \\
\hline & & $* *$ & & $.5<p<.75$ & & $* *$ & & $.95<p<.975$ & & ** & \\
\hline & & & ** & & $* *$ & & ** & & $.95<p<.975$ & & $* *$ \\
\hline & 1 & .2 & .3 & -1.8 & -5.1 & 7.8 & 9.3 & .1 & .2 & 7.1 & 8.3 \\
\hline & 2 & 3.9 & 4.6 & -1.9 & -5.2 & 7.8 & 8.9 & .4 & .6 & 7.2 & 8.2 \\
\hline$\approx$ & 3 & 3.5 & 4.0 & -1.9 & -5.2 & 7.8 & 8.9 & .4 & .6 & 7.3 & 8.3 \\
\hline for & 4 & 1.1 & 1.3 & -1.8 & -5.1 & 7.8 & 9.2 & .2 & .3 & 7.2 & 8.3 \\
\hline \multirow[t]{4}{*}{ II } & 5 & -23.0 & -28.9 & -1.8 & -4.0 & -2.6 & -2.7 & -1.2 & -1.3 & -3.4 & -3.5 \\
\hline & 6 & -16.4 & -22.1 & -1.7 & -3.1 & -2.4 & -2.5 & -1.2 & -1.3 & -3.5 & -3.6 \\
\hline & 7 & -15.5 & -21.2 & -1.6 & -2.4 & -4.1 & -4.2 & -1.2 & -1.4 & -4.5 & -4.7 \\
\hline & 8 & -11.2 & -13.3 & -1.8 & -3.7 & 1.0 & 1.0 & -1.2 & -1.3 & -1.0 & -1.0 \\
\hline \multirow[t]{3}{*}{$\chi^{2}$} & & 101 & 20206 & 3.6 & 47 & 112 & 1096 & 2.5 & 3.6 & 87 & 665 \\
\hline & & $* *$ & & $.75<p<.9$ & & $* *$ & & $.95<p<.975$ & & $* *$ & \\
\hline & & & ** & & $* *$ & & ** & & $.75<p<.9$ & & ** \\
\hline$z$ for & $\hat{\phi}$ & $121-$ & $\hat{\phi}_{2221}$ & & & & & -1.3 & -1.4 & & 1.2 \\
\hline
\end{tabular}

Note. For (A) through (E), the order of the two solutions for $\mathrm{z}$ is such that the loading for the 1st solution minus that for the 2nd one.

Uns.=Unstandardized variables, Sta. $=$ Standardized variables, $\mathrm{PF}=$ Principal factor solution, $\mathrm{NV}=$ Normal varimax solution, $\mathrm{RV}=\mathrm{Raw}$ varimax solution, $\mathrm{NQ}=$ Normal quartimin solution, NOV $=$ Normal oblique-varimax solution. The two asterisks stand for $p<.001$ for $\chi^{2}$ test with $d f=8$. 
Table 5: Correlation between factors for the eight physical variables

\begin{tabular}{|c|c|c|c|c|c|c|c|}
\hline & & & Uns. & Sta. & & Uns. & Sta. \\
\hline & & Estimates & $(\mathrm{SE})$ & ( SE ) & Estimates & $(\mathrm{SE})$ & $(\mathrm{SE})$ \\
\hline (A) & & & & Normal varimax & $\mathrm{x}$ solution & & \\
\hline Principal & & I & $\left(\begin{array}{ll}T & S\end{array}\right)$ & $(\mathrm{T} \quad \mathrm{S})$ & II & $\left(\begin{array}{ll}\mathrm{T} & \mathrm{S}\end{array}\right)$ & $\left(\begin{array}{ll}\mathrm{T} & \mathrm{S}\end{array}\right)$ \\
\hline factor & I & .78 & $\left(\begin{array}{ll}.031 & .031\end{array}\right)$ & $\left(\begin{array}{ll}.014 & .015\end{array}\right)$ & .62 & $\left(\begin{array}{ll}.039 & .040\end{array}\right)$ & $(.018 \quad .019)$ \\
\hline solution & II & -.62 & $\left(\begin{array}{ll}.039 & .040\end{array}\right)$ & $(.018 \quad .019)$ & .78 & $\left(\begin{array}{ll}.031 & .031\end{array}\right)$ & $(.014 \quad .015)$ \\
\hline (B) & & & & Normal varimax & $\mathrm{x}$ solution & & \\
\hline Raw & & I & $(\mathrm{T} \quad \mathrm{S})$ & $\left(\begin{array}{ll}\mathrm{T} & \mathrm{S}\end{array}\right)$ & II & $\left(\begin{array}{ll}T & S\end{array}\right)$ & $\left(\begin{array}{ll}T & S\end{array}\right)$ \\
\hline varimax & I & .9996 & $(.0005 .0005)$ & $(.0002 .0002)$ & .03 & $\left(\begin{array}{ll}.015 & .015\end{array}\right)$ & $\left(\begin{array}{ll}.006 & .006\end{array}\right)$ \\
\hline solution & II & -.03 & $(.015 \quad .015)$ & $(.006 \quad .006)$ & .9996 & $(.0005 .0005)$ & $(.0002 .0002)$ \\
\hline (C) & & & & Normal quartimi & in solution & & \\
\hline Normal & & I & $(\mathrm{T}$ & & II & $\left(\begin{array}{ll}T & S\end{array}\right)$ & \\
\hline varimax & I & .97 & $(.007 \quad .006)$ & & .24 & $\left(\begin{array}{ll}.026 & .026\end{array}\right)$ & \\
\hline solution & II & .24 & $\left(\begin{array}{ll}.026 & .026\end{array}\right)$ & & .78 & $(.007 \quad .006)$ & \\
\hline (D) & & & & Normal quartimi & in solution & & \\
\hline Raw & & I & $\left(\begin{array}{ll}T & S\end{array}\right)$ & $(\mathrm{T} \quad \mathrm{S})$ & II & $(\mathrm{T}$ & $(\mathrm{T}$ \\
\hline quartimin & I & .9999996 & .000003 .00000 & $9)(.000002 .00002)$ & .47 & $\left(\begin{array}{ll}.048 & .048\end{array}\right)$ & $\left(\begin{array}{ll}.048 & .048\end{array}\right)$ \\
\hline solution & II & .46 & $(.048 \quad .047)$ & $(.048 \quad .047)$ & .99994 & $(.00009 .0001)$ & $(.00009 .0001)$ \\
\hline (E) & & & & Normal quartimi & in solution & & \\
\hline Normal & & I & $(\mathrm{T} \quad \mathrm{S})$ & & II & $\left(\begin{array}{ll}\mathrm{T} & \mathrm{S}\end{array}\right)$ & \\
\hline oblique & I & .998 & $(.0005 .0004)$ & & .41 & $(.043 \quad .043)$ & \\
\hline $\begin{array}{l}\text { varimax } \\
\text { solution }\end{array}$ & II & .42 & $\left(\begin{array}{ll}.043 & .043\end{array}\right)$ & & .998 & $(.0005 .0005)$ & \\
\hline
\end{tabular}

Note. Uns.=Unstandardized variables, Sta. $=$ Standardized variables, $\mathrm{SE}=$ Standard errors, $\mathrm{T}(\mathrm{S})=$ Theoretical(Simulated) values, $\mathrm{I}(\mathrm{II})=$ FactorI(II).

When e.g., $p=20$ and $q=5,(41)$ is 460 . We have to take the inverse of the augmented matrix, though only once, and the method of taking the inverse of a matrix is an algebraic one without iterative approximation. Since usually only the submatrix $I^{*}$ in $I_{A}^{-1}$ is of interest, it is possible, if necessary, to reduce the problem size substantially by using the formula for the inverse of a partitioned matrix when $I$ is singular:

$$
I^{*}=J^{-1}-J^{-1} H\left(H^{\prime} J^{-1} H\right)^{-1} H^{\prime} J^{-1},
$$

where $J=I+H H^{\prime}$ and $H=\partial r^{\prime} / \partial \theta$ (see Silvey, 1975, p.178 and Browne, 1982, p.93; see Rao, 1973, 4i.1-2 for associated topics).

In the previous section for a numerical example, the chi-square tests were carried out separately for each pair of corresponding factors because each factor was a natural unit for testing the equalities of solutions. The comparison units can be changed if necessary. For instance, overall comparison between $\hat{B}_{1}$ and $\hat{B}_{2}$ may also be of interest. However, we should note that acov $\left(\operatorname{vec}\left(\hat{B}_{1}\right)\right)$ and $\operatorname{arov}\left(\operatorname{vec}\left(\hat{B}_{2}\right)\right)$ are singular when $\hat{B}_{1}$ and $\hat{B}_{2}$ are solutions in exploratory factor analysis. Among the elements of $\hat{B}_{1}$ and $\hat{B}_{2}$, at most $p q-\left(q^{2}-q\right) / 2$ and 
$p q-\left(q^{2}-q\right)$ elements give non-singular acovs for orthogonal and oblique factor models, respectively.

An additional application of the asymptotic covariances/correlations between $\hat{B}_{1}$ and $\hat{B}_{2}$ is the construction of the confidence intervals for functions of $\hat{B}_{1}$ and $\hat{B}_{2}$ (and estimated factor correlations) by using the asymptotic standard errors of the functions. For instance, Tucker's factor congruence coefficient, i.e.,

$$
\frac{\hat{\boldsymbol{b}}_{1(i)}^{\prime} \hat{\boldsymbol{b}}_{2(i)}^{\prime}}{\sqrt{\hat{\boldsymbol{b}}_{1(i)}^{\prime} \hat{\boldsymbol{b}}_{1(i)}} \sqrt{\hat{\boldsymbol{b}}_{2(i)}^{\prime} \hat{\boldsymbol{b}}_{2(i)}}},(i=1, \ldots, q),
$$

is one of the functions used in practice. The asymptotic standard error for (43) can be easily obtained by using the delta method. Ogasawara (1999b) derived the asymptotic standard error for (43) in the case when $\hat{B}_{1}$ and $\hat{B}_{2}$ are independent. However, since they are not independent in our case, the acovs between $\hat{B}_{1}$ and $\hat{B}_{2}$ should be considered. Tucker's coefficient for overall factor congruence can also be defined by using vec $\left(\hat{B}_{1}\right)$ and $\operatorname{vec}\left(\hat{B}_{2}\right)$. The asymptotic standard error for the overall congruence coefficient can be similarly obtained (the singularities for $\operatorname{acov}\left(\operatorname{vec}\left(\hat{B}_{1}\right)\right)$ and $\operatorname{acov}\left(\operatorname{vec}\left(\hat{B}_{2}\right)\right)$ are irrelevant for this case).

\section{REFERENCES}

Archer, C. O. \& Jennrich, R. I. (1973). Standard errors for rotated factor loadings. Psychometrika, 38, 581-592.

Browne, M. W. (1982). Covariance structures. In D. M. Hawkins (Ed.) Topics in applied multivariate analysis (pp. 72-141). Cambridge: Cambridge University Press.

Browne, M. W. \& Du Toit, S. H. C. (1992). Automated fitting of nonstandard models. Multivariate Behavioral Research, 27, 269-300.

Crawford, C. (1975). A comparison of the direct oblimin and primary parsimony methods of oblique rotation. British Journal of Mathematical and Statistical Psychology, 28, 201-213.

Cudeck, R. \& O'Dell, L. L. (1994). Applications of standard error estimates in unrestricted factor analysis: Significance tests for loadings and correlations. Psychological Bulletin, 115, 475-487.

Harman, H. H. (1976). Modern factor analysis (3rd ed.). Chicago: University of Chicago Press.

Jennrich, R. I. (1973a). Standard errors for obliquely rotated factor loadings. Psychometrika, 38, 593-604.

Jennrich, R. I. (1973b). On the stability of rotated factor loadings: The Wexler phenomenon. British Journal of Mathematical and Statistical Psychology, 26, 167-176.

Jennrich, R. I. (1974). Simplified formulae for standard errors in maximum likelihood factor analysis. British Journal of Mathematical and Statistical Psychology, 27, $122-131$.

Ogasawara, H. (1996). Standard errors for rotated factor loadings by normalized orthomax method. Japanese Journal of Behaviormetrics, 23(2), 122-129. (in Japanese with English abstract) 
Ogasawara, H. (1998a). Standard errors of several indices for unrotated and rotated factors. Economic Review, 49(1), 21-69, Otaru University of Commerce.

Ogasawara, H. (1998b). Standard errors for rotation matrices with an application to the promax solution. British Journal of Mathematical and Statistical Psychology, 51, 163-178.

Ogasawara, H. (1999a). Standard errors for the direct oblimin solution with Kaiser's normalization. Japanese Journal of Psychology, 70, 333-338. (in Japanese with English abstract)

Ogasawara, H. (1999b). Standard errors for matrix correlations. Multivariate Behavioral Research, 34, 103-122.

Rao, C. R. (1973). Linear statistical inference and its applications (2nd ed.). New York: Wiley.

Silvey, S. D. (1975). Statistical inference. London: Chapman \& Hall.

Received May 1999. Revised January 2000

\section{Appendix 1. Information Matrices}

1. Models for Unstandardized Manifest Variables

To obtain the $(i, j)$ th element of an information matrix (see $(7)$ ), we need $\partial \Sigma / \partial \theta_{i}$ and $\partial \Sigma / \partial \theta_{j}$. Some of the elements of information matrices may become simple by expanding the trace term. However, the expressions without expanding the trace are sufficient for exact computation.

In the following the lowercases denote the elements of the matrices denoted by the corresponding uppercases. The locations of the elements are denoted by the subscripts.

1) Two Sets of Orthogonally Rotated Solutions (see (4))

$$
\begin{aligned}
& \frac{\partial \Sigma}{\partial b_{1 s t}}=I_{s t} \Phi_{12} B_{2}^{\prime}, \frac{\partial \Sigma}{\partial b_{2 s t}}=B_{1} \Phi_{12} I_{t s} \\
& \frac{\partial \Sigma}{\partial \phi_{12 u v}}=B_{1} I_{u v} B_{2}^{\prime}, \frac{\partial \Sigma}{\partial \psi_{s}}=I_{s s} \\
& (s=1, \ldots, p ; t, u, v=1, \ldots, q)
\end{aligned}
$$

where $I_{s t}$ is the matrix of an appropriate size whose $(s, t)$ th element is one and others are zero.

2) An Orthogonally Rotated Solution and an Obliquely Rotated Solution (see (18))

$$
\begin{gathered}
\frac{\partial \Sigma}{\partial b_{1 s t}}=I_{s t} \Phi_{12} B_{2}^{\prime}, \frac{\partial \Sigma}{\partial b_{2 s t}}=B_{1} \Phi_{12} I_{t s}, \quad(s=1, \ldots, p: t=1, \ldots, q) \\
\frac{\partial \Sigma}{\partial \phi_{12 u v}}=B_{1}\left(2 I_{u v}+\Phi_{12} \Phi_{22}^{-1} I_{v u} \Phi_{12}\right) B_{2}^{\prime}, \quad(u, v=1, \ldots, q)
\end{gathered}
$$




$$
\begin{aligned}
\frac{\partial \Sigma}{\partial \phi_{22 u v}} & =-B_{1} \Phi_{12} \Phi_{22}^{-1}\left(I_{u v}+I_{v u}\right) \Phi_{22}^{-1} \Phi_{21} \Phi_{12} B_{2}^{\prime} \\
& =-B_{1} \Phi_{12} \Phi_{22}^{-1}\left(I_{u v}+I_{v u}\right) B_{2}^{\prime}, \quad(q \geq u>v \geq 1), \\
\frac{\partial \Sigma}{\partial \psi_{s}} & =I_{s s}, \quad(s=1, \ldots, p) .
\end{aligned}
$$

3) Two Scts of Obliquely Rotated Solutions (see (27))

$$
\begin{aligned}
\frac{\partial \Sigma}{\partial b_{1 s t}} & =I_{s t} \Phi_{12} B_{2}^{\prime}, \frac{\partial \Sigma}{\partial b_{2 s t}}=B_{1} \Phi_{12} I_{t s}, \quad(s=1, \ldots, p ; t=1, \ldots, q), \\
\frac{\partial \Sigma}{\partial \phi_{12 u v}} & =B_{1}\left(2 I_{u v}+\Phi_{12} \Phi_{22}^{-1} I_{v u} \Phi_{11}^{-1} \Phi_{12}\right) B_{2}^{\prime},(u, v=1, \ldots, q), \\
\frac{\partial \Sigma}{\partial \phi_{11 u v}} & =-B_{1} \Phi_{12} \Phi_{22}^{-1} \Phi_{21} \Phi_{11}^{-1}\left(I_{u v}+I_{v u}\right) \Phi_{11}^{-1} \Phi_{12} B_{2}^{\prime} \\
& =-B_{1}\left(I_{u v}+I_{v u}\right) \Phi_{11}^{-1} \Phi_{12} B_{2}^{\prime},(q \geq u>v \geq 1) \\
\frac{\partial \Sigma}{\partial \phi_{22 u v}} & =-B_{1} \Phi_{12} \Phi_{22}^{-1}\left(I_{u v}+I_{v u}\right) B_{2}^{\prime},(q \geq u>v \geq 1), \\
\frac{\partial \Sigma}{\partial \psi_{s}} & =I_{s s},(s=1, \ldots, p) .
\end{aligned}
$$

2. Models for Standardized Manifest Variables (see (34))

$$
\begin{aligned}
& \frac{\partial \Sigma}{\partial d_{i}}=I_{i i} P D+D P I_{i i},(i=1, \ldots, p), \\
& \frac{\partial \Sigma}{\partial \theta_{i}}=D \frac{\partial P}{\partial \theta_{i}} D
\end{aligned}
$$

where $\theta_{i}$ denote the parameters except $d_{1}, \ldots, d_{p}$ in (35). From (35), we see that the forms of $\frac{\partial P}{\partial \theta_{i}}$ are equivalent to (A1), (A2) and (A3), though their values are different unless $D=I_{p}$.

\section{Appendix 2. Properties of Transformation Matrices When Kaiser's Normalization is Employed}

Let a $p \times q$ factor loading matrix $\Lambda=\left(\lambda_{i j}\right)$ be rotated to $B$ by a rotation method with Kaiser's normalization. Let $H$ be the diagonal matrix with the $i$-th diagonal element being the square root of the communality of the $i$-th manifest variable (i.e., $\left.(H)_{i i}=\left(\sum_{j=1}^{q} \lambda_{i j}^{2}\right)^{1 / 2}\right)$. A rotation method with Kaiser's normalization transforms the rows of $\Lambda$ such that $\Lambda^{*}=H^{-1} \Lambda$. Then, $\Lambda^{*}$ is rotated to $B^{*}=\Lambda^{*} T$ by a transformation matrix $T$. The rotated loading matrix $B^{*}$ is 
denormalized as $B=H B^{*}=H \Lambda^{*} T=H H^{-1} \Lambda T=\Lambda T$. From this equation we find that $T$ is also a transformation matrix from $\Lambda$ to $B$, though it is determined by $\Lambda^{*}$ not by $\Lambda$. Then, we have the following proposition.

Proposition. Let $\hat{T}$ be a transformation matrix from an unrotated loading matrix $\hat{\Lambda}$ to a rotated loading matrix $\hat{B}$ by a rotation method with Kaiser's normalization, where $\hat{\Lambda}$ is a maximum likelihood estimate in an exploratory factor analysis model and the rotated factors are orthogonal or oblique. Then, $\hat{T}$ is independent of the scales of manifest variables.

Proof. Let $\hat{\Lambda}^{*}=\left(\hat{\lambda}_{i j}^{*}\right)$ be a row-normalized unrotated factor-loading matrix corresponding to the unrotated loading matrix $\hat{\Lambda}=\left(\hat{\lambda}_{i j}\right)$. Suppose that the standard deviation of the $i$-th manifest variable is multiplied by an arbitrary positive number $k$. Then, from the scale freeness of the maximum likelihood solution for an exploratory factor analysis model, we have $\tilde{\lambda}_{i j}=\hat{k} \hat{\lambda}_{i j}$, where $\hat{k}$ is the estimate of $k$ and a parameter with tilde denotes the estimate after the scale transformation. Since $\bar{\lambda}_{i j}^{*}=\hat{k} \hat{\lambda}_{i j} /\left\{\sum_{j=1}^{q}\left(\hat{k} \hat{\lambda}_{i j}\right)^{2}\right\}^{1 / 2}=\hat{\lambda}_{i j} /\left\{\sum_{j=1}^{q}\left(\hat{\lambda}_{i j}\right)^{2}\right\}^{1 / 2}=\hat{\lambda}_{i j}^{*}$, we have $\tilde{\Lambda}^{*}=\hat{\Lambda}^{*}$. By applying this result to other manifest variables, we see that $\tilde{\Lambda}^{*}\left(=\hat{\Lambda}^{*}\right)$ is independent of the scales of the $p$ manifest variables. From this and the fact that $\hat{T}$ is determined by $\hat{\Lambda}^{*}$, the result of the proposition follows.

Using the proposition we have the following corollary.

Corollary. Let $\hat{\Phi}_{12}, \hat{\Phi}_{11}$ and $\hat{\Phi}_{22}$ be the maximum likelihood estimates of the parameters in the previous sections. Suppose that they are obtained by rotation methods with Kaiser's normalization. Then, the covariances of the estimates of the parameters in $\Phi_{12}, \Phi_{11}$ and $\Phi_{22}$ are independent of the scales of manifest variables.

Proof. Let $\hat{T}_{1}$ and $\hat{T}_{2}$ be the estimates of the transformation matrices with Kaiser's normalization. From the result of the above proposition $\hat{T}_{1}$ and $\hat{T}_{2}$ are independent of the scales of the manifest variables. Since $\hat{\Phi}_{12}=\hat{T}_{1}^{\prime} \hat{T}_{2}, \hat{\Phi}_{11}=$ $\hat{T}_{1}^{\prime} \hat{T}_{1}$ and $\hat{\Phi}_{22}=\hat{T}_{2}^{\prime} \hat{T}_{2}$, these matrices and consequently their covariances are independent of the scales of the manifest variables.

The result of the corollary suggests a nice property of Kaiser's normalization. 